\title{
Médiévales
}

Langues, Textes, Histoire

72 | printemps 2017

Roman du Genji et société aristocratique au Japon

\section{Lire et écrire le Genji aujourd'hui}

Reading and Writing the Genji Today

\section{Anne Bayard-Sakai}

\section{(2) OpenEdition}

Journals

Édition électronique

URL : https://journals.openedition.org/medievales/8111

DOI : 10.4000/medievales. 8111

ISSN : $1777-5892$

\section{Éditeur}

Presses universitaires de Vincennes

\section{Édition imprimée}

Date de publication : 22 juin 2017

Pagination : 145-154

ISBN : 978-2-84292-612-0

ISSN : 0751-2708

\section{Référence électronique}

Anne Bayard-Sakai, «Lire et écrire le Genji aujourd'hui », Médiévales [En ligne], 72 I printemps 2017, mis en ligne le 28 février 2019, consulté le 23 avril 2022. URL : http://journals.openedition.org/medievales/ 8111 ; DOI : https://doi.org/10.4000/medievales.8111 


\section{Anne Bayard-Sakai}

\section{Lire et écrire le Genji aujourd'hui}

Parmi les événements éditoriaux de la dernière décennie au Japon, l'un des plus remarquables est la publication d'une Anthologie de la littérature japonaise (Nihon bungaku zenshû), compilée sous la houlette de l'écrivain Ikezawa Natsuki ${ }^{1}$. Le terme zensh $\hat{u}$, que l'on trouve dans le titre de cette anthologie et dont la traduction littérale serait «série complète », recouvre un type de publication qui a joué un rôle essentiel dans la modernisation de l'industrie éditoriale japonaise à partir du milieu des années 1920. Si certaines de ces séries correspondent à des œuvres complètes d'écrivains, d'autres sont des anthologies couvrant un domaine : contrairement à ce que suggère le terme zensh $\hat{u}$, il ne s'agit donc pas pour ces séries d'être exhaustives, mais d'offrir un large accès à un genre (il existe ainsi plusieurs zenshû de littérature policière) ou encore à la production littéraire d'une époque comme le propose, par exemple, un zenshû de la littérature de l'ère Shôwa (1926-1989). Ajoutons qu'il existe des zensh û dans d'autres domaines que littéraires, puisqu'on trouve des anthologies, par exemple, de textes philosophiques. Quoi qu'il en soit, ces séries ont soutenu le développement de l'édition moderne au Japon; elles ont très régulièrement alimenté les rayonnages des librairies et, si l'offre a été si abondante, il faut souligner qu'elle répond à une demande à la fois institutionnelle (celle par exemple des bibliothèques) et individuelle, les lecteurs étant d'autant plus incités à lire plusieurs ouvrages de ces séries que leur lancement s'accompagne généralement d'une offre promotionnelle couvrant l'ensemble des volumes.

Toutefois, la publication de ces séries a connu un net fléchissement à partir des années 1970 et surtout 1980, fléchissement qui reflète la crise de l'édition et la transformation des pratiques de lecteurs de plus en plus

1. Anthologie publiée par la maison d'édition Kawade shobô shinsha. 
tournés vers des choix ponctuels que satisfont les collections de poche bon marché. L'annonce par la maison d'édition Kawade shobô shinsha de la parution de deux anthologies, une première de littérature mondiale à paraître entre 2007 et 2011, puis une deuxième de littérature japonaise dont la publication a débuté fin 2014, avait donc été accueillie avec un certain scepticisme. Or, contrairement à toute attente, ces séries ont connu un grand succès, les 30 volumes de la première série ayant ainsi été vendus à plus de 400000 exemplaires $^{2}$. Quant à la deuxième anthologie, dont 24 volumes sur les 30 prévus sont parus à ce jour, elle avait atteint en novembre 2016 un chiffre de vente cumulé de 350000 exemplaires $^{3}$.

Laissons de côté ici le succès de la première anthologie, et ce qu'elle révèle du rapport du Japon à la world literature, pour nous intéresser à la deuxième. Sa programmation annonce, pour 2017, trois volumes consacrés au Genji monogatari. Une nouvelle apparition donc de ce classique sur la scène littéraire japonaise ? Certes, mais elle prend sens dans un contexte spécifique, qui touche à la fois la question générale de la présence de la littérature classique au Japon aujourd'hui, et la place singulière qui revient au Genji monogatari dans la culture contemporaine.

\section{Du classique, mais à travers du moderne}

Si cette anthologie de littérature japonaise est atypique, elle ne l'est pas seulement pour être un objet éditorial incongru à une époque où les anthologies semblent appartenir à un passé révolu. Elle l'est également pour au moins deux autres raisons. Elle couvre, d'une part, toute l'histoire de la littérature japonaise depuis ses origines jusqu'à nos jours, alors qu'en règle générale, jusqu'à présent, les anthologies respectaient la distinction entre les littératures dites classique et moderne ; d'autre part, elle propose les textes classiques non pas dans leur version originale, mais traduits en langue moderne par des écrivains contemporains. Pour comprendre cette distinction et ce souci de traduction, il faut rappeler que la littérature classique japonaise est écrite soit en sino-japonais, soit dans une langue d'écriture qui s'élabore entre le VIII et le $\mathrm{IX}^{\mathrm{e}}$ siècle, la littérature moderne se développant, quant à elle, à partir des années 1880 lorsque s'invente une nouvelle langue d'écriture qui rapproche langues orale et écrite, le vernaculaire et le scripturaire. Si la distinction des littératures classique et moderne est aussi clairement marquée, le fait tient d'abord à la différence des langues concernées : sans un apprentissage, relativement poussé, du lexique et plus encore de la syntaxe de la langue classique, un lecteur d'aujourd'hui ne peut comprendre un texte ancien. Les anthologies précédentes rassemblant

2. Chiffre donné par le journal Sankei (édition du 21 février 2015).

3. Chiffre donné par le journal Asahi (édition du 2 novembre 2016). 
des textes classiques les présentent donc annotés, voire très annotés, et généralement accompagnés d'une traduction en langue moderne établie par un spécialiste du domaine.

On comprend donc par contraste la singularité du choix effectué pour cette dernière anthologie, et ses présupposés, dont le premier est l'affirmation que la continuité historique l'emporte sur la séparation des corpus classique et moderne ${ }^{4}$. Mais cette affirmation n'a d'effet éditorial que si les textes sont accessibles aux lecteurs sur un mode que l'on pourrait dire homogénéisé, leur permettant de s'y rapporter d'une seule et même manière : tous les textes sont donc proposés en langue moderne, y compris les textes classiques qui ne sont pas publiés en version originale mais en traduction. L'objectif ici n'est pas de renforcer la connaissance des textes en tant que classiques, mais de faciliter une lecture contemporaine pour un public, le plus large possible, de non-spécialistes. Mais comment atteindre cet objectif sans consentir un effort supplémentaire afin de rendre les textes attrayants ?

Pour y parvenir, les traductions des textes classiques ont été confiées, non pas à des universitaires, mais à des écrivains occupant une place importante, autrement dit très visible, sur la scène littéraire japonaise d'aujourd'hui. Le sous-entendu évident - et que nous ne discuterons pas ici - est qu'un écrivain, romancier, poète, est plus à même de produire une traduction séduisante, dotée de qualités littéraires indiscutables, qu'un universitaire ; le résultat, non moins évident, est que les volumes de textes classiques traduits par des écrivains sont placés sur le même plan que ceux consacrés aux écrivains modernes et contemporains. Mais l'effet indirect tout aussi important est que l'entreprise provoque la mise en commun, ou plus précisément le cumul des légitimités ; la valeur symbolique et canonique attachée à un classique en tant que classique s'ajoute à la renommée d'un auteur contemporain célèbre - à sa valeur symbolique, certes, mais aussi à ses potentialités commerciales, son côté bankable, sa capacité à mobiliser son propre lectorat, et en retour cet auteur gagne en légitimité en produisant une traduction d'un texte dont la canonicité n'est pas contestable ${ }^{5}$.

Ce que confirme ainsi en réalité l'anthologie est une certaine manière qu'a la littérature moderne japonaise de se rapporter à son passé et d'en tirer parti. Car, si l'anthologie exhibe les liens entre classique et moderne par le biais des traductions et systématise les transferts de légitimité qu'elles provoquent, ces liens ne sont pas explorés pour la première fois. Pour en rester

4. Ikezawa Natsuki, le maître d'œuvre de l'anthologie, souligne dans une déclaration liminaire à la publication les éléments de continuité qui définissent la littérature japonaise. $C f$. le site de la maison d'édition Kawade Shobô shinsha: http://www.kawade.co.jp/nihon_ bungaku_zenshu/\#schedule.

5. La sélection des textes classiques proposés par l'anthologie ne ménage aucune surprise. 
dans le cadre des $\mathrm{XX}^{\mathrm{e}}$ et $\mathrm{XXI}^{\mathrm{e}}$ siècles, nombreux sont les textes classiques traduits en langue moderne par les écrivains modernes et contemporains, nombreuses aussi les œuvres dérivées des textes classiques proposées par les auteurs d'aujourd'hui. Et plus le texte de départ est canonique, plus les productions dérivées prolifèrent, cette prolifération renforçant bien entendu en retour sa canonicité. On ne s'étonnera donc pas de constater que, dans toute cette production «palimpsestique », le Genji monogatari occupe la première place.

\section{Variations sur le Genji}

Dans toute la littérature japonaise, le Genji monogatari est probablement le seul texte à avoir suscité l'apparition d'un genre second, celui des traductions de ce texte par des romanciers. Depuis la version due à la grande poétesse Yosano Akiko publiée en 1912 jusqu'à celle de Setouchi Jakuchô qui paraît en 1997, en passant par celles de Tanizaki Jun. ichirô publiées pour la première entre 1939 et 1941, pour la deuxième entre 1951 et 1954, ou celle de Enchi Fumiko qui paraît entre 1972 et 1973, ces traductions ont permis au Genji monogatari de toucher un public pour qui la version originale est devenue illisible, et aux auteurs-traducteurs de capter une part du prestige attaché à un texte occupant une position centrale dans l'histoire culturelle du pays ${ }^{6}$. La traduction en langue moderne du roman, due à l'écrivaine Kakuta Mitsuyo et annoncée dans les volumes à paraître en 2017 de l'Anthologie de la littérature japonaise, va donc venir s'ajouter à une liste déjà relativement fournie. Mais ces traductions, à la fois intra-textuelles et, en quelque sorte, intra-littéraires, ne sont pas les seules réalisations hypertextuelles dérivées du Genji. L'importance reconnue très tôt au roman explique la succession d'œuvres parodiques qui émaillent l'histoire de la littérature classique ${ }^{7}$. Mais plus intéressant pour nous ici est le fait qu'aujourd'hui encore sont publiés de très nombreux textes dont le Genji monogatari est la source, dans une relation de dérivation qui s'affiche. Nous ne saurions ici relever tous ceux qui ont pu paraître, ne fût-ce que depuis le début du $\mathrm{XXI}^{\mathrm{e}}$ siècle, et nous arrêterons donc sur deux recueils, parus l'un en 2007, l'autre en 2008, qui semblent représentatifs du travail de réécriture déployé à partir du Genji monogatari.

Le premier point à souligner concerne la concomitance de date de parution des deux ouvrages. En 2008 a en effet été célébré avec faste et

6. Pour une analyse détaillée, $c f$. A. BAYARD-SAKAI, «Texte et prétexte : traduire le Genji en langue moderne », Cipango, numéro hors-série «Autour du Genji monogatari », Paris, 2008, p. 155-185.

7. Sur ce point, $c f$. par exemple H. SHIRANE éd., Envisioning The Tale of Genji : Media, Gender, and Cultural Production, New York, 2008 ; M. EMMERICH, The Tale of Genji : Translation, Canonization and World Literature, New York, 2013. 
sous l'égide d'un comité d'organisation officiel placé sous le patronage d'organismes publics tels que le ministère de la Culture ou la municipalité de Kyôto, le millénaire de l'écriture du Genji monogatari - le choix de cette année s'appuyant, de manière lâche, sur des mentions puisées dans le Journal de Murasaki Shikibu (Murasaki Shikibu nikki), rédigé entre 1008 et 1010 . Une quantité d'événements de tous genres furent organisés à l'époque, allant de la parution de numéros spéciaux de revues à la mise en vente de gâteaux commémoratifs, et la licence du logo officiel de la célébration aurait été vendue plus de 155 millions de fois ${ }^{8}$. On comprend alors aisément que le monde éditorial ait voulu profiter de cette occasion, et les deux ouvrages en question, comme d'autres, sont des produits de circonstance. Une différence essentielle distingue pourtant les deux: l'un, Genji monogatari Kokonotsu no hensô (Neuf variations sur le Genji monogatari), rassemble des textes de neuf écrivains différents, alors que l'autre, Yomichigae Genji monogatari (Le Genji monogatari en lectures décalées), comprend huit textes d'un même auteur.

Si les Neuf variations sur le Genji monogatari sont connues aujourd'hui, en édition de poche, sous ce nom, elles ont été initialement publiées sous le titre Nain Sutôrîzu obu Genji, où l'on aura reconnu la transposition dans le système phonétique japonais d'un Nine Stories of Genji ${ }^{9}$. Le recueil, sur le plan de l'édition, est un curieux objet, dans la mesure où, contrairement aux habitudes, il ne porte aucun nom de responsable, comme si l'idée de rassembler ces textes allait de soi, comme s'il s'agissait d'une génération éditoriale spontanée induite par la frénésie Genji que suscitait l'opération du millénaire. En outre, la version de poche ne comporte pas de postface, contrairement ici encore aux habitudes puisqu'en général une postface (le plus souvent allographe) accompagne les ouvrages publiés en poche, expliquant les circonstances d'écriture ou de composition de l'ouvrage, et en proposant un commentaire. Le titre, en anglo-japonais, détonne, et est clairement destiné à capter l'attention du lecteur. La distance temporelle qui sépare l'écriture du Genji de ces textes est ainsi mise en scène et métaphorisée en une distance langagière. Et pourtant, lorsque l'ouvrage passe en collection de poche, le titre retenu, celui des Neuf variations, a perdu toute sa charge de provocation. Aucune explication de ce changement n'est fournie au lecteur, qui en est réduit aux conjectures, la raison la plus plausible étant peut-être que le titre anglojaponais prêtait à confusion, nourrissant chez le lecteur l'attente de textes plus décalés que ceux proposés en réalité.

8. Chiffre donné par M. EMmerich, The Tale of Genji..., p. 13. Selon ses calculs, ces événements ont totalisé plus de 156 millions de participants.

9. Nain sutôrîzu obu Genji (Nine Stories of Genji), Tôkyô, 2008 ; version en collection de poche sous le titre Genji monogatari Kokonotsu no hensô (Neuf variations sur le Genji monogatari), Tôkyô, 2011. 
Ces neuf «variations » sont donc écrites chacune par un auteur différent. Tous sont des écrivains reconnus, lauréats de prix littéraires majeurs, occupant une place importante sur la scène littéraire. On voit donc fonctionner, comme dans le cas de l'anthologie de littérature japonaise mentionnée plus haut, ce double transfert de légitimité, les auteurs bénéficiant du prestige du Genji, tandis que le Genji se voit conférer une modernité et une actualité du fait d'être repris dans ces variations contemporaines dues à des écrivains largement lus. Comment alors chacun de ces textes se définit-il ? Adoptant le format d'une nouvelle longue de 25 à 50 pages, tous prélèvent un fragment, un épisode, une ligne narrative du roman-fleuve qu'est le Genji monogatari, pour en présenter une autre version. Sans entrer ici dans une analyse détaillée des neuf textes, il faut souligner les différences de stratégies adoptées par les auteurs.

Certains, en effet, restent proches du texte de départ, se contentant de résumer un livre, en gardant un mode d'énonciation assez proche de l'original - c'est le cas par exemple de la première nouvelle, où la romancière Matsuura Rieko reprend le livre «Hahakigi », «L'arbre-balai » ${ }^{10}$, développant surtout les séquences que l'on dira les plus romanesques; d'autres en revanche choisissent de prendre la plus grande distance possible - c'est le cas par exemple de l'écrivaine Kanehara Hitomi qui reprend le livre «Aoi », «Les mauves », en transposant dans le monde contemporain l'histoire, qui se réduit à celle d'une grossesse vue par un jeune couple portant les mêmes noms personnels que les personnages du Genji ; les sept autres enfin se situent à des distances variables de ces deux pôles. On notera d'ailleurs avec intérêt que les lecteurs ne réagissent pas de la même manière face à l'éloignement ou la proximité du texte de départ. Les commentaires laissés sur les sites de lecture par des lecteurs anonymes montrent que certains sont rebutés par les nouvelles qui reposent sur des transpositions radicales, que ce soit de l'histoire, du mode narratif, ou du style : ils sont rebutés, en d'autres termes, quand ils ne retrouvent pas le texte d'origine. D'autres, à l'opposé, critiquent les nouvelles jugées trop proches de l'original, comme si elles ne respectaient pas les règles du jeu : ils s'interrogent sur l'intérêt de réécrire un texte si ce n'est pas pour s'en éloigner.

Mais quelles que soient les réactions des lecteurs, un fait demeure : les textes n'existent que comme hypertextes et tout est fait, d'un point de vue éditorial, pour qu'il en soit ainsi. Sans parler du titre général de l'ouvrage, qui rend en somme impossible pour le lecteur de lire les nouvelles hors du

10. Toutes les versions françaises des termes et des noms présents dans le roman sont reprises de la traduction du texte proposée par René Sieffert : MURASAKI SHIKIBU, Le Dit du Genji, trad. R. SIEFFERT, Paris, 1988. Le livre «L'arbre-balai » apparaît aussi sous le titre de « L'arbre-mirage ». 
paradigme Genji, chaque nouvelle reprend le titre d'un livre du texte de départ. Mieux, pour chaque nouvelle, la page de titre est suivie d'une autre portant un résumé du livre correspondant dans l'original. L'ensemble du dispositif est donc destiné à amener le lecteur, sinon même à le contraindre, à procéder à une lecture hypertextuelle. La question qui demeure ouverte est alors la suivante : le lecteur dispose-t-il d'un critère autre que la distance au texte d'origine pour apprécier les nouvelles ? Peut-il s'affranchir du dispositif contraignant pour déployer une lecture autonome? Il serait alors manifestement en infraction avec les règles rigides qu'impose la matérialité même de l'objet livre qu'il a entre les mains.

\section{Parodier le Genji}

Venons-en alors au Yomichigae Genji monogatari (Le Genji monogatari en lectures décalées), que 1'on doit à Shimizu Yoshinori, écrivain connu comme spécialiste de l'écriture parodique ${ }^{11}$. Le terme yomichigae, traduit ici par «lectures décalées», est une invention de l'auteur. Il est composé du verbe yomи, «lire», et de chigae, lecture irrégulière des caractères du verbe tagaeru, qui porte l'idée de ne pas faire coïncider quelque chose, d'engager quelque chose dans une mauvaise voie, d'errer. Autrement dit, par ce titre, Shimizu suggère qu'il propose certes des lectures du Genji, mais dont le principe est qu'elles ne coïncident pas avec l'original, tout leur intérêt se trouvant dans le décalage - ce qui, on l'aura compris, est le principe même de la parodie. Le schéma adopté par l'auteur est ainsi de reprendre, dans chacune des huit nouvelles du recueil, des épisodes célèbres du Genji monogatari et de leur faire subir un déplacement significatif. Ainsi, la première nouvelle, intitulé «Yûgao satsujin jiken», «L'affaire de l'assassinat de Yûgao », est une nouvelle policière, dans laquelle une sorte de Watson écoute une sorte de Sherlock Holmes lui démontrer que le personnage de Yûgao, Belle-du-soir, ne meurt pas victime d'un sort jeté par une maîtresse du Prince Genji consumée par la jalousie, contrairement à ce que semble indiquer le Genji monogatari. Ou encore, dans la nouvelle «Puraido» (dont le titre est calqué sur l'anglais pride, « orgueil »), la jalousie de la Dame de la Chambre de la Sixième Avenue, Rokujô no miyasudokoro, à l'égard d'une jeune maîtresse du Prince, est transformée pour devenir le ressentiment d'une actrice vieillissante à l'égard d'une jeune actrice qui lui a ravi les faveurs de son amant. Autre exemple encore, la relation que le Prince Genji entretient avec l'innocente et quelque peu niaise Suetsumuhana, La fleur dont se cueille la pointe, que caractérise son long nez rougeoyant, est transposée au Texas, à Jacksonville, pour

11. SHIMIZU Y., Yomichigae Genji monogatari (Le Genji monogatari en lectures décalées), Tôkyô, 2007. 
devenir «Rôzubaddo », «Rosebud », l'histoire d'amour entre le playboy John Cosby et Cathy, la jeune fille méprisée de tous parce que niaise, mal habillée, et dotée d'un nez démesuré et rougeoyant.

Il est sans doute inutile de multiplier les exemples : Shimizu s'amuse ici à jouer sur de nombreux facteurs de variation, temporels, géographiques, génériques, mais en prenant soin de laisser suffisamment d'indices pour que la parodie soit toujours reconnue comme telle. Une fois encore, le titre même du recueil contraint la lecture, qui peut difficilement s'affranchir de la référence au texte d'origine. En outre, le titre de chaque nouvelle est complété par le nom du personnage du Genji qui est parodiquement mis en scène dans le texte ${ }^{12}$. De même, les jeux onomastiques rappellent constamment les liens entre chacune des histoires racontées et le Genji, puisque les noms des personnages rappellent ceux des personnages du roman (à l'exception - et pour cause - de «Rosebud ${ }^{13}$ ). En dehors de ces indices, la capacité à apprécier véritablement la qualité du travail de réécriture parodique dépend, évidemment, de la connaissance que l'on a du roman. C'est d'ailleurs ce que remarquent les lecteurs dans les réactions publiées sur les sites, certains, manifestement, ayant un souvenir assez précis du Genji pour se délecter des variations proposées par Shimizu, alors que d'autres regrettent de n'avoir qu'une idée trop lointaine du roman pour tirer vraiment plaisir de la lecture des nouvelles.

Mais, sans doute, tous les lecteurs de Shimizu savent d'emblée à quoi ils ont affaire : des parodies. À la différence des nouvelles reprises dans les Neuf variations, la distance par rapport à l'hypotexte ne saurait, sous peine d'absurdité, être considérée comme un défaut. Plus l'amplitude du déplacement sera importante, mieux l'objectif parodique sera donc atteint, même si demeure entière la question de la qualité intrinsèque du texte (à supposer que cette question ait un sens).

Des traductions en langue moderne aux réécritures, parodiques ou non, on ne peut que constater que le Genji continue à alimenter une production hypertextuelle considérable. Le phénomène, il faut au demeurant le souligner, dépasse de loin le seul registre de la littérature : on a ainsi pu parler d'une « culture Genji » (Genji bunka), constituée de tous les produits

12. Pour être précis, dans la table des matières qui ouvre le recueil, seul le titre de chaque texte est indiqué ; ensuite, chaque fois qu'un titre est redonné en ouverture d'une nouvelle, il s'accompagne, dans une police de taille légèrement inférieure, du nom du personnage du Genji concerné. On peut en inférer que Shimizu joue à la fois sur la distance et la proximité du texte d'origine.

13. Sauf à considérer que le bouton de rose en question rappelle la « fleur dont on cueille la pointe » qui donne son nom au personnage du Genji. On nous pardonnera de laisser ici de côté la question d'une éventuelle référence à Orson Welles. 
dérivés du Genji monogatari - films, films d'animation, jeux vidéo, mangas, images, vêtements, etc. - qui jouit d'un incontestable degré d'autonomie et d'achèvement par rapport au texte original ${ }^{14}$. Mais une question demeure alors non résolue, celle de la connaissance du texte premier.

La version originale du Genji, disions-nous, est illisible pour un lecteur d'aujourd'hui. Les traductions en langue moderne, surtout celles proposées par des écrivains de renom, ont pu être très lues - les dix volumes de la traduction Setouchi Jakuchô publiée en 1997 se sont vendus au total à plus de 2,2 millions d'exemplaires ${ }^{15}$. Un savoir détaillé de la trame du roman a pu également être acquis par les lecteurs du manga Asaki yume mishi (Ayant fait de vains rêves) de Yamato Waki, publié dans différents magazines de 1979 à 1993, et dont les volumes des différentes éditions se sont vendus à plus de 18 millions d'exemplaires ${ }^{16}$. Le développement enfin de cette culture Genji autonomisée, renforcée lors du millénaire par des phénomènes de vulgarisation et de diffusion de connaissances même vagues sur le Genji, prouve en tout état de cause qu'il existe bien un savoir approximatif du Genji qui irrigue largement le corps social.

De quoi alors ce savoir est-il fait? Sans doute d'une idée très générale de la trame, de souvenirs des épisodes marquants, tandis que demeure probablement inaperçu tout ce qui tient à la littérarité même du texte, par exemple sa richesse stylistique ${ }^{17}$. Il apparait dès lors que tous ces phénomènes de réécriture évoqués ici partagent en définitive une approche assez schématique de l'œuvre originale, dans la mesure où ils en reprennent uniquement les faits saillants - la prolifération de ces réécritures amenant paradoxalement le Genji monogatari à cesser d'exister comme texte pour ne plus être qu'un hypotexte. Peut-être est-ce pour un classique la seule manière d'exister de nos jours, peut-être convient-il de s'en attrister - mais le Genji, aujourd'hui, demeure certainement un cas rare où l'œuvre venue

14. $C f$. KomachiYa T., « Genji bunka no genzai » (La culture Genji aujourd'hui), dans II H. et KURATA M. éd., Gendai bunka to Genji monogatari (La culture contemporaine et le Genji monogatari), Tôkyô, 2008, p. 10. Dans une autre contribution figurant dans le même ouvrage, TATEISHI Kazuhiro signale même l'existence de ce qu'on pourrait appeler un mode de comportement qui, dans l'univers internet des otaku (des fanatiques de mangas, de jeux vidéo, etc.), porte le nom de Hikaru Genji keikaku «projet Hikaru Genji », du nom du héros du roman, et se trouve marqué par la volonté pygmalionesque de façonner conformément à ses désirs une très jeune fille, comme le fait le prince Genji avec Wakamurasaki, la Jeune Grémil (TATEISHI K., « Genji monogatari kanren shuppan to kaishaku kyôdôtai » (Les publications relatives au Genji monogatari et les communautés interprétatives), dans II H. et KURATA M. éd., Gendai bunka to Genji monogatari..., p. 167.

15. Chiffre donné sur la page suivante $:$ http://news.mynavi.jp/news/2000/10/11/06.html.

16. Chiffre donné par le journal Yomiuri dans son édition du 3 avril 2007.

17. «Le Genji monogatari, en lui-même, n'est pas tellement lu; ce qui circule en réalité, ce sont des résumés de l'histoire, des figures de personnages réduits à des types, et des connaissances portant sur les mœurs ou l'environnement général de l'époque Heian » (TATEISHI K., « Genji monogatari kanren shuppan to kaishaku kyôdôtai »..., p. 157). 
du passé, ce qu'il est d'abord, continue à produire des variantes, des versions autres, réussissant ainsi, malgré tout, à être un texte d'aujourd'hui.

Anne Bayard-Sakai - Inalco/Centre d'Études Japonaises (CEJ) Institut Universitaire de France

\section{Lire et écrire le Genji aujourd'hui}

Si les lecteurs japonais d'aujourd'hui ne peuvent lire les textes classiques en version originale, ils y ont accès à travers des œuvres dérivées, rédigées en langue moderne, parmi lesquelles, en premier lieu, les traductions. De nouveaux projets éditoriaux systématisent ce mode de diffusion des textes classiques, proposant des traductions dues à des écrivains reconnus. Dans le cas du Genji monogatari, ces traductions intra-langagières ne sont qu'un des aspects de la prolifération de textes dérivés, réécritures, parodies, transpositions de toutes sortes, dont on analyse ici quelques caractéristiques, afin de déterminer comment un texte du passé, canonique, peut exister dans le Japon d'aujourd'hui.

Canonisation - littérature japonaise - hypertexte - intertextualité - Japon traduction

\section{Reading and Writing the Genji Today}

The contemporary readers of Japanese literature are unable to read the classical texts in their original version, and can only reach them through derivative versions, written in modern language, such as translations. Thus, new publishing projects are built, offering access to classical texts through translations provided by well-known writers. In the case of the Genji monogatari, those intralinguistic translations are but one of the numerous phenomenon related to the proliferation of derivative texts, rewritings, parodies, transpositions of different kinds. Some of the main features of those derivative texts are analysed here, in order to determine how a canonical text can exist in Japan today.

Canonization - Hypertext - Intertextuality - Japan - Japanese Literature Translation 